\title{
Synthetic biology: advancing biological frontiers by building synthetic systems
}

\author{
Yvonne Y Chen ${ }^{1,2,}$, Kate E Galloway ${ }^{3,+}$ and Christina D Smolke*
}

\begin{abstract}
Advances in synthetic biology are contributing to diverse research areas, from basic biology to biomanufacturing and disease therapy. We discuss the theoretical foundation, applications, and potential of this emerging field.
\end{abstract}

Synthetic biology is an emerging field of interdisciplinary research that seeks to transform our ability to probe, manipulate, and interface with living systems by combining the knowledge and techniques of biology, chemistry, computer science, and engineering. Its main aim is to increase the ease and efficiency with which biological systems can be designed, constructed, and characterized. Core efforts in the field have focused on the development of tools to support this goal, including new approaches to biological design and fabrication. Although the first generation of synthetic systems demonstrated genetic circuits that encode dynamic behavior, cellular computational operations, and biological communication channels, more recent research has focused on implementing synthetic biological devices and systems in diverse applications, including disease therapy, environmental remediation, and biosynthesis of commodity chemicals. As the field matures, synthetic biology is advancing biological frontiers by expanding biomanufacturing capabilities, developing next-generation therapeutic approaches, and providing new insights into natural biological systems. Here, we review the theoretical foundations, diverse tool kits, and engineered systems that have emerged from synthetic biology and discuss current as well as potential future applications, which include in-depth studies of basic biology (such as understanding endogenous signaling pathways and

\footnotetext{
'These authors contributed equally to this work

*Correspondence: csmolke@stanford.edu

${ }^{4}$ Department of Bioengineering, Stanford University, 473 Via Ortega, MC 4201,

Stanford, CA 94305, USA

Full list of author information is available at the end of the article
}

feedback circuits) and new frontiers in health and medicine (such as identification of diseased cells and targeted therapeutics).

\section{Conceptual frameworks for biological design}

A central aim of synthetic biology is to increase the ease and efficiency with which biological systems can be designed, constructed, and characterized. Although the manipulation of biological organisms and molecular pathways long preceded the emergence of synthetic biology, the engineering of biological systems has been a largely ad hoc exercise. A main reason is that biology is inherently diverse, mutable, and context specific. Natural biological substrates, including genetic elements such as promoters and genes, do not always behave predictably when implemented in different combinations, and details such as how individual parts are physically connected can vary widely across different construction methods. As a result, the components designed and assembled for one biological system often cannot be predictably reused in another system. Synthetic biology seeks to address this challenge by implementing a more 'engineering-ready' conceptual framework that emphasizes the need to generate and report biological constructs in a manner that is conducive to their understanding and utilization by a broad community of researchers.

The application of engineering tools such as abstraction, decoupling, and standardization was proposed early in the emergence of synthetic biology to support the efficiency and scaling of the biological system design process [1]. An abstraction hierarchy that dissects the engineering process into several design levels - DNA, parts, devices, and systems - provides synthetic biologists with a means to manage complexity and distribute tasks. The design process at each abstraction level can be performed relatively independently of the other levels, and detailed information critical to one abstraction level need only be considered by designers operating at that level. This division of labor reduces the amount of information that each designer must be expert in to successfully design a part, device, or system.

Decoupling refers to the strategy of partitioning a complicated problem into simpler tasks that can be 
tackled separately and assembled into a complete solution. The separation of design and fabrication processes is an important example of decoupling supported by advances in design tools and fabrication platforms. The increasing efficiency and decreasing cost of DNA synthesis allow synthetic biologists to design novel systems with the confidence that DNA components can be readily synthesized by commercial sources. Furthermore, advances in DNA sequencing and synthesis provide researchers with access to biological components encoding functions of interest using sequence information deposited in databases, eliminating the need for physical exchange of genetic materials.

Standardization takes several forms, including standardization of physical assembly, functional assembly, and characterization/measurements. Early physical assembly standards used biological parts flanked by standardized sequences, enabling the interchangeable combination and sequential assembly of parts conforming to the specified standard through a constant restriction-enzyme/ligationmediated cloning strategy $[2,3]$. Significantly less progress has been made in the field on functional assembly standards, which focus on identifying sequence interfaces between two types of parts (for example, ribosome binding site (RBS) and gene) that allow functional coupling and predictable activity, independent of the specific sequence of each part. Several early physical-assembly strategies encountered obstacles because the proposed standards impaired the functional assembly of parts by requiring the insertion of standard sequences between each part. In response, the field is shifting to assembly methods that do not require restriction-enzymemediated cloning $[4,5]$. Finally, technical measurement and reporting standards have been proposed to eliminate discrepancies that result from disparate experimental methods and to provide more reliable and thorough characterization data [6]. Standardized characterization data will support reliable sharing and reuse of parts, devices, and systems such that new designs can build on the foundation of previous work and move beyond the $a d$ hoc model of system development.

\section{Advances in fabrication methods for genetic systems}

As synthetic biological systems become increasingly sophisticated, fabrication methods with larger capacities, greater precision, higher speed, and lower cost have become increasingly important. Outpacing the development of novel parts and devices, a number of groundbreaking fabrication techniques have been demonstrated in recent years, allowing researchers to focus on system design while outsourcing or performing system fabrication with higher efficiencies than was previously possible. Advances in multiplex oligonucleotide synthesis and assembly with microfluidic arrays have allowed cheaper de novo synthesis of gene-length fragments [7-9]. Furthermore, several techniques have been developed for the assembly of large DNA fragments, moving the field beyond laborious and time-consuming molecular cloning.

For example, transformation-associated recombination (TAR) in the yeast Saccharomyces cerevisiae has been used to construct yeast artificial chromosomes encoding genes and pathways isolated from several different organisms $[4,10]$. Yeast artificial chromosomes can be further modified with bacterial artificial chromosome sequences to transfer the constructs to bacteria and subsequently to mammalian cells [11]. Enzymatic in vitro assembly methods, such as one-step isothermal DNA assembly, can allow DNA molecules of several hundred kilobases to be assembled without restriction-enzymemediated digestion [5,12]. A combination of in vitro and TAR-based assembly methods was used to assemble and clone the first bacterial genome from chemically synthesized oligonucleotides [13]. However, large sets of parts encoding similar functions with distinct sequences are needed to avoid undesired recombination events between components that share similar sequences when assembling large genetic systems with recombination-based strategies.

In addition to DNA synthesis and assembly, methods have been developed for high-throughput genome modification. Multiplex automated genome engineering (MAGE) uses the bacteriophage $\lambda$-Red single-strandedDNA-binding protein $\beta$ to achieve allelic replacement in Escherichia coli. This process can greatly accelerate the optimization of biological systems and metabolic pathways, provided that the target genes are known and that an efficient screening method is in place to identify the desired variants within the diverse libraries generated [14]. An alternative method termed trackable multiplex recombineering (TRMR) has been developed to support applications in which a priori knowledge of which target gene to modify is lacking, enabling rapid mapping of genes and quantification of population dynamics [15]. A complementary technology called hierarchical conjugative assembly genome engineering (CAGE), which has been used to combine portions of a genome that have been modified by MAGE, was also recently described [16]. Although genome modification has been reported in yeast [17], most high-throughput methods have been limited to demonstrations in E. coli and the extension of these technologies to mammalian cells remains an important challenge.

\section{Constructing the toolbox: synthetic biological parts and devices}

Over the past decade, the synthetic biology community has built a large collection of biological parts and devices 
Table 1. The synthetic biology toolbox: common components used in synthetic biological systems

\begin{tabular}{|c|c|c|}
\hline & Component & Function \\
\hline \multirow[t]{2}{*}{ Transcriptional } & Constitutive promoter libraries $[19,20]$ & Provide continuously ON gene expression at pre-determined levels \\
\hline & $\begin{array}{l}\text { Inducible promoters (for example, responsive to tetracycline, } \\
\text { IPTG, gaseous acetaldehyde [22], or light [23]) }\end{array}$ & $\begin{array}{l}\text { Provide conditional and, in certain cases, titratable gene expression } \\
\text { in response to inducer signal }\end{array}$ \\
\hline \multirow[t]{3}{*}{ Posttranscriptional } & $\begin{array}{l}\text { Non-coding regulatory RNAs [28] (such as riboregulators } \\
{[29,30] \text {, ribozyme switches }[31,51] \text {, and RNAi switches }[32,33] \text { ) }}\end{array}$ & $\begin{array}{l}\text { Control protein production levels by regulating mRNA stability or } \\
\text { translation initiation in response to molecular input }\end{array}$ \\
\hline & Alternative splicing modulators [35] & $\begin{array}{l}\text { Control protein production levels or protein activity by regulating } \\
\text { alternative splicing of mRNA in response to molecular input }\end{array}$ \\
\hline & RNase substrate libraries [80] & $\begin{array}{l}\text { Control protein levels through tunable hairpin elements that direct } \\
\text { transcript cleavage }\end{array}$ \\
\hline \multirow[t]{2}{*}{ Posttranslational } & Degradation tags $[24,25]$ & Modulate protein levels by shortening protein half-lives \\
\hline & Split inteins $[26,27]$ & $\begin{array}{l}\text { Provide biosensing and modulate protein activity by conditionally } \\
\text { splicing inactive protein fragments together into functional wholes }\end{array}$ \\
\hline
\end{tabular}

IPTG, isopropyl- $\beta$-D-thio-galactoside; RNAi, RNA interference.

with diverse functions [18] (Table 1). One focus has been the development of control devices that alter protein levels in response to prescribed input signals, supporting the ability to reprogram cellular behavior. Most systems demonstrated so far use transcription-based control, incorporating constitutive and inducible promoters from a small set of well-characterized parts, such as the tetracycline- and isopropyl- $\beta$-D-thio-galactoside (IPTG)inducible promoters. However, inadequacies in these often-used promoters, including inducer toxicity and limited selection of input choices and response profiles, have motivated the development of libraries of constitutive promoters with varying strengths $[19,20]$ and the generation of new inducible promoters responsive to small molecules [21], gaseous acetaldehyde [22], and light [23]. The expanding collection of promoter parts increases the range of stimuli and expression levels that can be achieved, providing greater flexibility in controlling the behavior of biological systems.

As the diversity of gene regulatory processes in natural biological systems comes to light, efforts have also been directed to developing control devices that act through posttranscriptional and posttranslational mechanisms. In addition to parts such as degradation tags [24,25] and split inteins [26,27], non-coding regulatory RNAs have been used to construct a number of control devices [28]. In one example, microbial gene expression was regulated by engineered RNA-responsive regulators (termed 'riboregulators') that modulate translation initiation by either obstructing or releasing the RBS of a target gene in response to the presence of a separately transcribed RNA sequence [29]. Researchers have demonstrated the utility of riboregulators in a variety of applications, including protein localization studies, perturbation of stress response networks, and programmable cell killing [30].
RNA-based devices responsive to small-molecule and protein inputs have also been demonstrated, exerting control over both transgenic and endogenous protein expression in bacteria, yeast, and mammalian cells [31-33], leading to applications ranging from bacteriamediated detection and breakdown of pesticides [34] to disease-marker detection and cell-fate regulation in mammalian cells [35]. The unique properties of RNAbased control devices - including ease of design and construction, small genetic footprint, high energy efficiency, fast regulatory time scales, and the ability to tailor input responsiveness and regulatory stringency - have made RNA a versatile substrate for designing programmable control systems.

In addition to controlling protein levels, synthetic biologists have developed tools to modulate the spatial organization of protein molecules inside cells, resulting in new strategies for regulating or rewiring cellular activities encoded in metabolic and signaling pathways [36]. In one example, researchers constructed synthetic feedback loops within the yeast mating mitogenactivated protein (MAP) kinase pathway by recruiting modulator proteins to the pathway scaffold protein Ste5 through fusing leucine zipper domains to each component, and demonstrated circuits with pulse generator, accelerator, delay, and ultrasensitive switch functions $[37,38]$. In another example, synthetic protein scaffolds that spatially recruit metabolic enzymes were implemented in E. coli, enabling the stoichiometric optimization of three mevalonate biosynthetic enzymes and achieving a 77-fold increase in product titer while avoiding cellular toxicity caused by the accumulation of a pathway intermediate [39]. As an alternative to protein-based scaffolds, rationally designed RNA strands have recently been shown to assemble into higher-order structures, 
including sheets and nanotubes, inside bacterial cells [40]. An RNA scaffold was applied to a two-enzyme hydrogen biosynthesis pathway and shown to increase hydrogen production by up to 48 -fold compared with an unscaffolded system [40]. These examples highlight the utility of spatial engineering in enhancing and modifying biological pathways.

\section{Synthetic gene circuits}

One of the hallmarks of synthetic biology has been the drive to engineer biological systems from the bottom up. Model-driven design of synthetic gene circuits has demonstrated the ability to build circuits of specified function [41-43]; differences between models and realized circuits have illuminated important and unique aspects of biological system behavior, such as the effects of degradation processes, cooperativity, and noise [44-46]. In addition to inspiring the design of more robustly operating systems, the insights gained through synthetic approaches have contributed to our understanding of natural biological systems [47].

\section{Genetic circuits encoding dynamic behaviors}

The construction of two genetic circuits encoding dynamic behavior, the repressilator [44] and the toggle switch [48], marked two early efforts in genetic circuit design in synthetic biology. The repressilator, a three-ring oscillator built from a loop of three repressor-promoter pairs, demonstrated that an oscillatory response could be generated from biological parts not found in natural biological oscillators. However, the synthetic oscillator showed fluctuations and heterogeneity not observed in natural circadian oscillators, which generally use interlinked positive and negative feedback loops [45]. Subsequent generations of oscillators have interlinked positive and negative transcriptional feedback loops of different strengths to drive more robust oscillatory dynamics with tunable periods and amplitudes in bacterial [42] and mammalian cells [49] (Figure 1a). In the post-genomics era, the importance of precise timing and coordination of gene expression in regulating systems-level behavior is increasingly appreciated. Well-defined, synthetic gene circuits that control the temporal profile of gene expression can elucidate the contributions of expression dynamics to natural time-dependent processes, such as cell signaling, cell-fate determination, and development. Synthetic gene circuits also offer the potential to develop genetically encoded strategies for intervening and controlling these natural processes.

\section{Encoding cellular logic and computing functions}

Genetic circuits that perform computations and logical evaluations of cellular information provide the ability to assess intracellular states and environmental signals.
They transmit this information into changes in cellular function, such as production of easily assayed readouts, activation of metabolic pathways, or initiation of cell-fate decisions. Towards this goal, genetic circuits and devices capable of performing logical evaluations have been built to detect small molecules (using tandem promoter systems [50] and RNA devices [51]), and small RNAs such as small interfering (si)RNAs (using tandem RNA interference (RNAi) target sites [52]) (Figure 1b). These various schemes have demonstrated the classic NOT, OR, NOR, and AND gates that are used to build larger logic evaluators and computations.

Methods for counting and maintaining memory of system states will enable a broader spectrum of intracellular computing. A genetic circuit that can count up to three exposure events to a small-molecule inducer was built in bacteria by nesting polymerase-promoter pairs controlled by riboregulators responsive to an inducible transactivator [53]. Although this system captured brief induction pulses, system performance was highly dependent on pulse duration and frequency. The incorporation of genetic memory offers an alternative strategy to increase the robustness of counting events over longer time frames. A three-event counter circuit was demonstrated by using DNA recombinase-based cascades that record each event as a permanent change to the DNA, where the output of each recombinase event would 'prime' the next promoterrecombinase pair in the circuit [53]. Synthetic networks of feedback loops have been built as memory circuits that lock a system in one state through sustained production of proteins following a transient signal that initiates the state. For example, toggle switches engineered to show bistability in bacteria [48] and mammalian cells [54] use architectures of mutually inhibitory feedback loops to achieve reversible memory of small-molecule pulses. As another example, a positive feedback loop built from a synthetic transcriptional activator cascade demonstrated heritable memory over many generations in yeast [55].

One recurrent limitation in adapting biological systems to perform computation through the rules of binary logic is the analog nature of the responses. In particular, gene expression leakage in the OFF state can contribute to improper input processing and high basal output, diminishing an evaluator's signal-to-noise ratio $[48,53,55]$. In addition, control of highly lethal proteins and proteins that mediate irreversible genetic changes requires stringent OFF states. To address this issue, researchers have layered transcriptional and posttranscriptional control elements within genetic circuits to provide strategies for achieving stringent regulation of transgenes in mammalian [56,57] and bacterial cells [30]. In one example, an inducible promoter was layered with repressible expression of a small hairpin (sh)RNA to achieve undetectable expression levels of the highly lethal 


\section{(a) Dynamic Circuits}

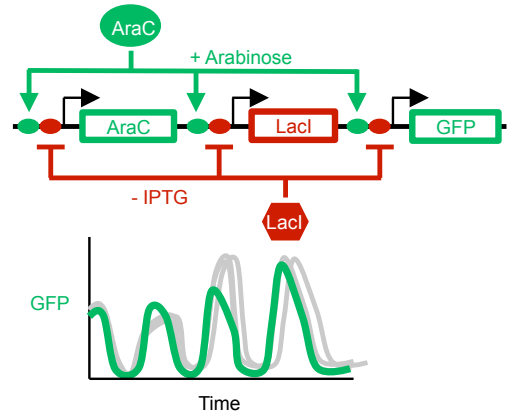

(b) Logic Circuits
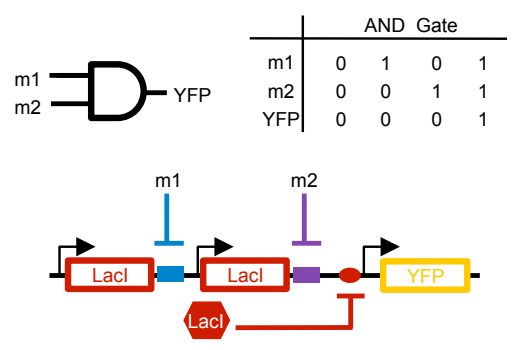

(c) Communication Circuits

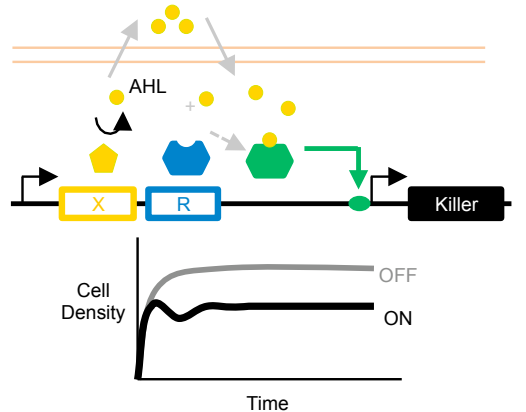

\section{(d) Dynamic + Communication Circuits = Synchronized Quorum of Genetic Clocks \\ (e) Logic + Communication Circuits = Edge Detector}
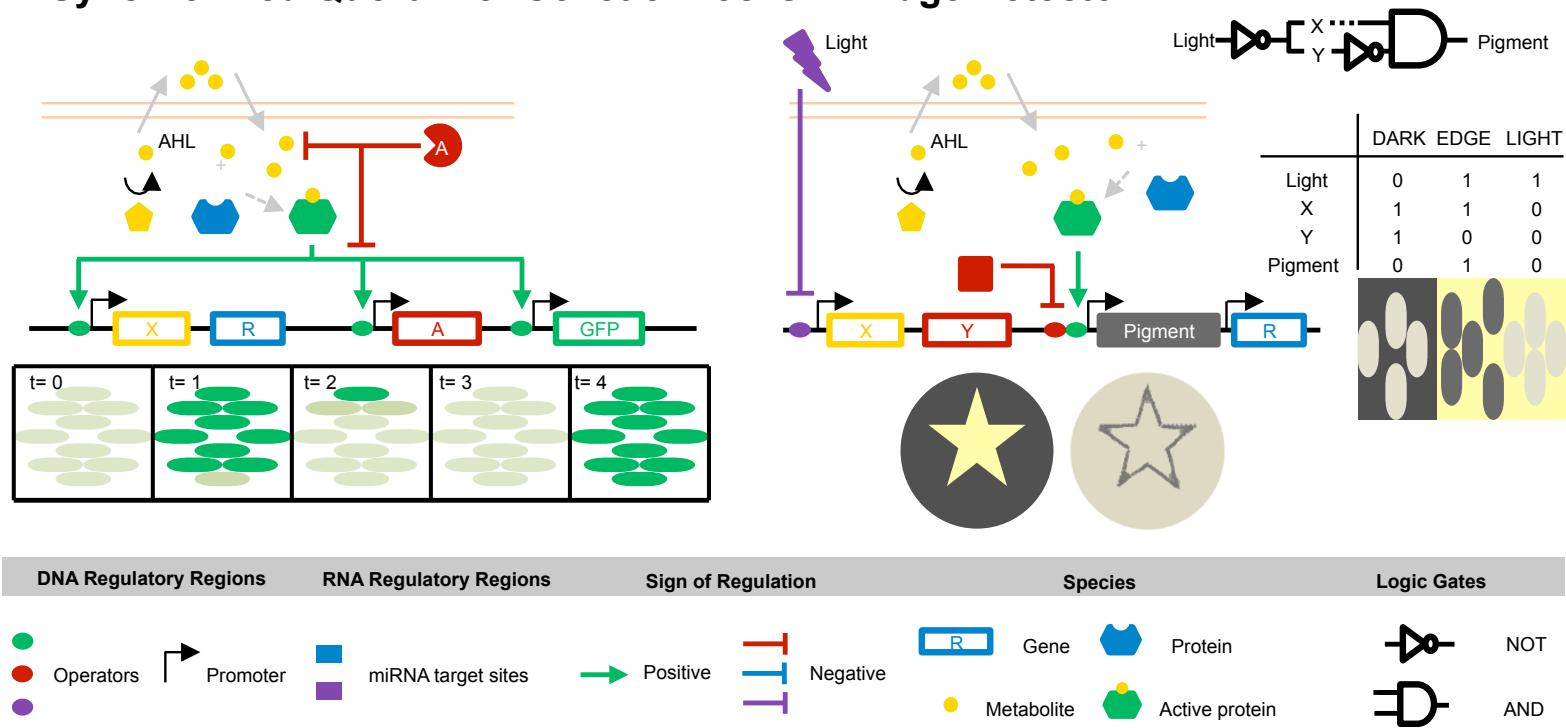

Figure 1. Synthetic circuits that perform diverse functions can be coupled to achieve higher-order responses. (a) Interlinked positive and negative feedback loops of different strengths drive an oscillatory response. Arabinose-responsive transcriptional activator (AraC) expression positively modulates gene expression and results in a positive feedback loop, whereas the isopropyl- $\beta$-D-thio-galactoside (IPTG)-responsive inhibitor of the lac promoter (Lacl) inhibits expression and generates a negative feedback loop. The small-molecule inducers arabinose and IPTG modulate the strength of these feedback loops [42]. GFP, green fluorescent protein. (b) A mammalian AND gate composed of RNA interference (RNAi) target sites evaluates small interfering (si)RNA inputs. Unique RNAi target sites are placed in the $3^{\prime}$ UTR of two lacl genes, and Lacl regulates the expression of a fluorescent reporter, resulting in an AND logic evaluator for the siRNA inputs $m 1$ and $m 2$ [52]. YFP, yellow fluorescent protein. (c) Quorumsensing circuitry allows population control. Cell density is broadcast by the diffusible small molecule acyl-homoserine lactone (AHL), which is synthesized by the enzyme LuxI (X). As cell density and AHL concentration increase, LuxR (R), a transcriptional regulator, binds AHL and initiates expression of a'killer' gene (encoding CcdB, a lethal protein that targets the DNA gyrase complex), ultimately reducing the steady-state cell density [58]. (d) Interlinking positive and negative feedback loops with communication circuitry enables oscillation synchronization across a population of cells. Expression of R positively regulates expression of X, R, GFP, and AiiA (A), an enzyme that degrades AHL. As A increases in concentration, it degrades AHL and negatively modulates protein expression levels [62]. (e) Combining logic processing with communication circuitry enables a synthetic biological edge detection system. The expression of $X$ and the transcriptional repressor $\mathrm{Cl}(\mathrm{Y})$ is turned $\mathrm{ON}$ in cells in the dark region, where $Y$ represses the expression of the pigment-producing protein (pigment: $\beta$-galactosidase, an enzyme that cleaves a substrate to produce a black pigment). However, diffusion of AHL synthesized by cells in the dark region activates $\mathrm{R}$ in cells at the edge of the light region (where $Y$ is turned OFF), thus turning ON expression of pigment only in cells along this edge [63].

diphtheria toxin in the OFF state, thus enabling induced cell death only in the ON state [56]. Although tight OFF states are desirable for binary computing, biological computing necessarily exploits the analog and tunable nature of gene expression. Connecting logical circuit outputs to changes in cellular state requires the ability to 
both identify thresholds of expression at which cellular behavior diverges and tune the output to cross that threshold when triggered. Combining the computational ability of logical evaluators with improved strategies for leakage minimization and output tuning should enable more robust computing. These tools can expand our ability to detect and treat diseases by increasing diagnostic certainty and improving precision in gene expression, and can also be used to probe previously inaccessible information sets, such as the temporal and spatial profiles of particular developmental genes, which will inform our fundamental understanding of biology.

\section{Communication circuits supporting more complex behaviors}

Communication systems are required to coordinate events and tasks between different cells in a population. Synthetic communication circuits have been engineered in bacteria using various bacterial quorum-sensing systems. In these systems, a lactone signal is broadcast with increasing strength as cell density increases. At a given threshold level, lactone binds and activates a transcriptional regulator, upregulating the expression of a target gene. Broadcasting and receiving can be incorporated within a single cell population or distributed between 'sender' and 'receiver' cells. Incorporating both functions in a single population programmed to regulate a killer gene resulted in population control and demonstrated how population heterogeneity can be exploited to achieve a robust population response [58] (Figure 1c). Segregating tasks and localizing the sender population established a radial gradient of signaling molecules. Coupling the quorum-sensing circuitry to a band-pass circuit, which detects a specified range of input concentrations, achieved formation of various radial patterns in the receiver cells [59]. In addition, connecting bacterial quorum systems to synthetic circuits has demonstrated dual-population consensus response and symbiosis in biofilms [60,61] and synchronized genetic clocks [62] (Figure 1d). Finally, coupling a light-responsive device [23] to logic-processing circuitry and a communication module resulted in a biological edge detector [63] (Figure 1e). These examples demonstrate how synthetic circuits can distribute and coordinate computational tasks across a population of cells to achieve complex responses similar to what is observed in natural pattern formation and development.

Beyond bacterial systems, mammalian receiver cells have been engineered to respond to volatile chemical signals [64] and metabolic conditions [65] using engineered synthetic promoters. These receivers can potentially be paired with various processing circuits and sender cells to generate synthetic hormone-signaling systems and synthetic ecosystems. The eventual coupling of metabolic functions and cell-fate circuitry to synthetic hormonesignaling systems will enable spatial patterning of cell differentiation and timing of coordinated cellular responses, a requisite for complex tissue formation and function.

\section{Moving towards real-world applications}

Despite remarkable advances in the design and construction of increasingly sophisticated genetic circuits over the past decade, the transition of these systems to real-world applications has been constrained by the limited availability of devices that can connect synthetic circuitry with information in living systems. However, synthetic biologists are developing new ways to connect natural and engineered systems. For example, exploiting existing connections between synthetic circuitry and intracellular information, researchers have used the natural correlation between DNA damage and proteolysis of the $\mathrm{ON}$ state inhibitor $\lambda \mathrm{cI}$ in a genetic toggle switch to record transiently induced DNA damage through the formation of a biofilm [66]. Taking another approach, researchers have constructed synthetic sensor devices from natural components, such as promoter-repressor pairs $[67,68]$, signaling pathway components [69], and small RNAs and their target sites $[52,57]$, to extract information from biological systems. As the range of sensor devices, processing circuitry, and output modules expands, synthetic biology is poised to address a broad scope of biological, medical, and biotechnological challenges.

\section{Understanding biology by building}

The ability to construct synthetic genetic circuits from the bottom up has enabled researchers to approach questions in basic biology from a new angle. Instead of perturbing natural pathways or model systems, biological systems can be probed by constructing artificial circuits and examining their behavior and interactions with endogenous machinery [70]. For example, in one investigation into why a particular circuit architecture was selected over others that produced similar dynamics, researchers compared the native Bacillus subtilis circuit that regulates differentiation into the competence state (when cells can take up DNA from the environment) with an engineered circuit [71] (Figure 2a). Modeling and empirical results both indicated that, despite generating excitable dynamics with similar shapes and frequency to those of the native competence circuit, the engineered circuit achieved greater precision in the duration of competence. It was further demonstrated that the greater variability in the native circuit conferred an advantage in the form of more consistent transformation efficiency over a large range of extracellular DNA concentrations, highlighting the effective exploitation of noise in natural circuits [71]. Additional synthetic circuits have been constructed to study the modularity and plasticity of 


\section{(a) Understanding Biology}

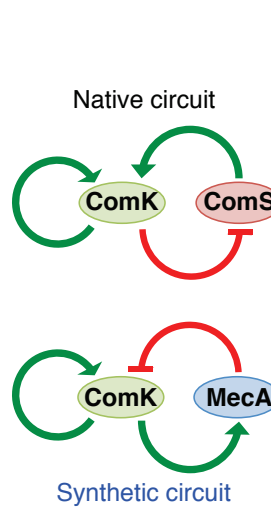

Similar competence dynamics
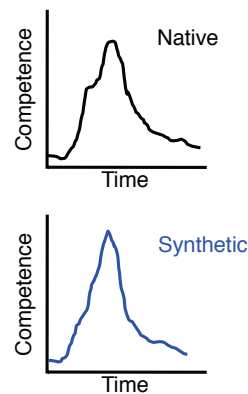

Differing precision in competence duration
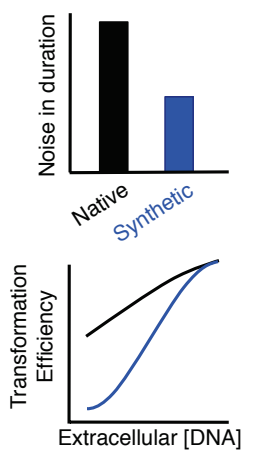

Differing consistency in transformation efficiency

\section{(b) Biomanufacturing}
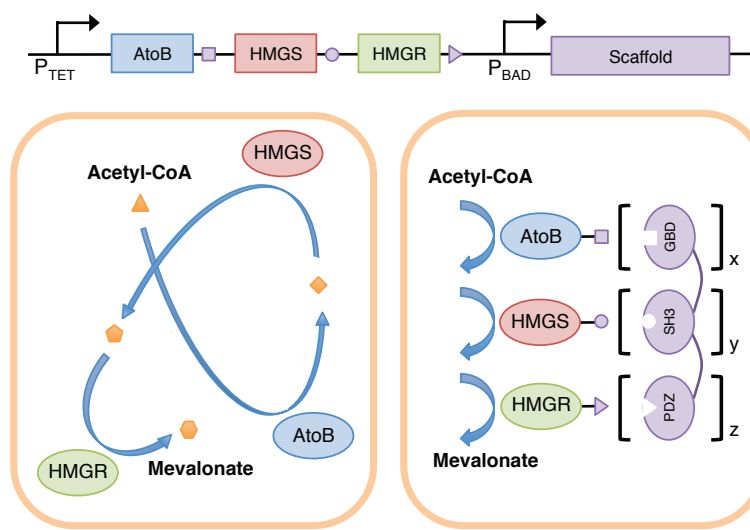

Unscaffolded pathway mevalonate production

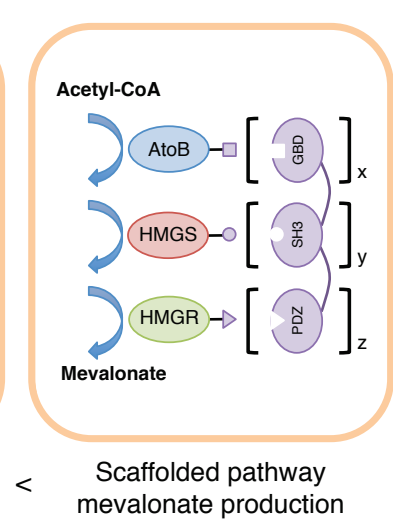

\section{(c) Therapeutics and Diagnostics}

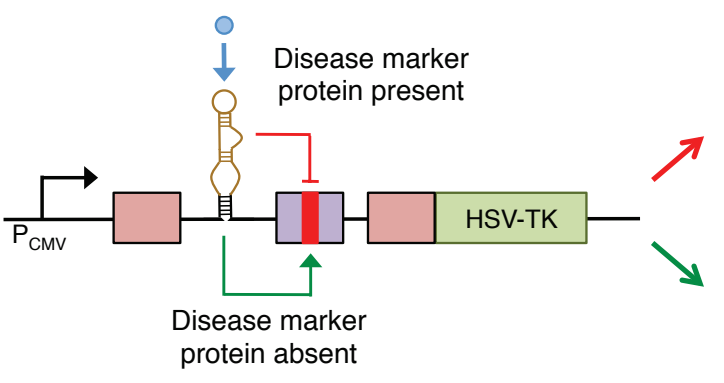

Disease marker
protein absent
Spliced transcript excludes exon containing stop codon

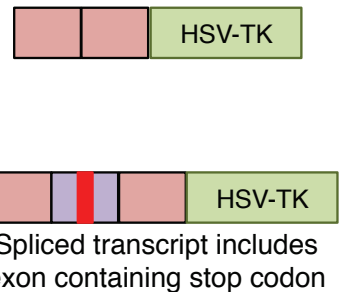
exon containing stop codon
Suicide-gene expression leads to death of diseased cells

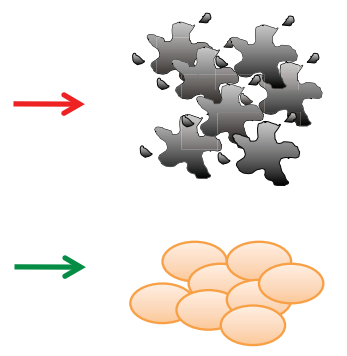

Suicide-gene expression suppressed in non-diseased cells

Figure 2. Synthetic biological circuits can aid in understanding of biology, improve biomanufacturing productivity, and enable diseasetargeted therapy. (a) The native circuit regulating competence in B. subtilis was compared with a synthetic circuit with similar dynamics to reveal architecture-specific variability in the duration of competence and consequent differences in the consistency of transformation efficiency over large ranges of DNA concentration [71]. (b) A synthetic protein scaffold was used to increase the biosynthesis of mevalonate from acetylCoA in E. coli. The scaffold consists of three protein-protein interaction domains (GBD, the GTPase binding domain from the actin polymerization switch N-WASP; SH3, the Src homology 3 domain from the adaptor protein CRK; and PDZ, the PSD95/DlgA/Zo-1 domain from the adaptor protein syntrophin) in various copy numbers connected by glycine-serine linkers. Pathway enzymes (AtoB, acetoacetyl-CoA thiolase; HMGS, hydroxymethylglutaryl-CoA synthase; HMGR, hydroxymethylglutaryl-CoA reductase) were each fused to the ligands of one interaction domain and recruited to the protein scaffold [39]. $P_{\text {TET, }}$ tetracycline-inducible promoter; $P_{B A D^{\prime}}$ arabinose-inducible promoter. (c) $A$ targeted therapeutic circuit was constructed by inserting an RNA aptamer near an alternatively spliced exon harboring a stop codon in a three-exon, two-intron minigene fused to herpes simplex virus thymidine kinase (HSV-TK). Binding of a disease marker protein to the aptamer results in exclusion of the alternative exon, expression of a suicide gene, and killing of diseased cells [35]. CMv' cytomegalovirus promoter.

MAP kinases [72,73] and the interaction between Notch and Delta critical for cell patterning during development [74], among others [70], illustrating the utility of synthetic biology in expanding our fundamental understanding of biology.

\section{Expanding biomanufacturing capabilities}

Biomanufacturing is one of the more compelling and immediate applications of biotechnology that promises sustainable synthesis strategies for alternative energy sources, commodity chemicals, and high-value specialty chemicals such as therapeutic drugs. A major challenge of biosynthetic pathway engineering lies in balancing the levels and activities of the many heterologous pathway enzymes to achieve optimized productivity and yield of desired compounds in the microbial host. Synthetic biology is transforming biosynthesis capabilities by providing new tools that support pathway construction and 
optimization. For example, researchers have recently combined TAR-based assembly strategies with sets of biosynthetic pathway parts (including enzyme coding regions, promoters, and terminators) to demonstrate onestep, whole-pathway assembly for a variety of naturalproduct pathways [75-77]. In another example, combinatorial libraries of tunable intergenic regions (TIGRs) harboring a number of RNA regulatory elements, including terminators, RNase cleavage sites, and stabilizing hairpins, were assembled in the non-coding regions between three heterologous enzymes in the mevalonate biosynthetic pathway expressed from a polycistronic transcript in E. coli. Researchers screened library variants for the TIGR sequences that resulted in optimal relative expression levels of each enzyme to increase mevalonate production; the best mevalonate producers decreased accumulation of a toxic intermediate and increased growth rate [78]. Libraries of modular control elements, including promoters and RNA regulatory elements, that have broad ranges of predictable activities have also been generated $[19,79]$. Recently, a library of RNase cleavage elements was used in yeast to titrate a key enzyme and thus flux through the endogenous ergosterol pathway, which competes with synthetic terpenoid pathways for the common precursor farnesyl pyrophosphate [80]. Finally, several new tools supporting colocalization of heterologous enzymes, such as protein- and RNA-based scaffolds, are being used to develop pathway optimization strategies based on spatial engineering [39,40] (Figure 2b).

\section{Advancing next-generation therapeutics and diagnostics}

By developing new strategies to interface with and manipulate natural biological systems, synthetic biology holds exciting promise in developing new therapeutic approaches. For instance, synthetic biologists are developing genetic circuits that link therapeutic activities to the detection of molecular disease signals to develop targeted therapeutics with increased efficacy and safety. In one example, a layered microRNA (miRNA)- and transcription-factorbased logic circuit was used to distinguish a cervical cancer cell line (HeLa) from other cell lines based on the detection of a unique miRNA profile [57]. Positive identification of HeLa cells through this logic circuit was subsequently linked to either expression of a reporter protein, as a model diagnostic device, or expression of a protein that led to cell death as a model therapeutic device. In another example, to restrict cell death to diseased cells showing hyperactive signaling, researchers developed protein-responsive RNA devices that could detect increased signaling through the NF- $\mathrm{KB}$ and Wnt pathways and transmit this information into changes in the expression of a clinically relevant suicide gene that sensitizes cells to an apoptosis-inducing prodrug [35] (Figure 2c). These types of autonomous sense-and-control circuits offer potential applications in the long-term surveillance and intervention of chronic diseases, such as gout and diabetes [68,81]. Circuits currently under development that link genetic targets to clinicianmodulated external inputs will provide an unprecedented level of temporal and spatial control over complex therapeutic activities. For example, systems have been described that support light-modulated glucose homeostasis [82] and drug-modulated control over in vivo gene expression [83] and T-cell proliferation [84].

\section{Where will synthetic biology take us?}

The biological parts, genetic circuits, and fabrication techniques that have been developed and continue to be improved on offer exciting potential in diverse applications, from environmental engineering to regenerative medicine. Synthetic biological systems capable of detecting, reporting, and/or removing hazardous substances have been reported [85-88], and their implementation in robust host organisms suitable for environmental release will provide a new paradigm for environmental remediation. In the area of health and medicine, synthetic intercellular communication systems that regulate spatial patterning, timing of coordinated cellular responses, and tissue homeostasis have the potential to make significant contributions to tissue engineering. Furthermore, synthetic control circuitry may reduce the inherent tumorigenicity of stem cells [89] and improve the efficiency of induced pluripotent stem cell reprogramming [90]. Novel genetic circuits capable of guiding the ex vivo construction of complex tissues may be built in the foreseeable future as researchers continue to unravel the systems biology behind cell-fate decisions [91,92].

Efforts in synthetic biology so far have covered a wide range of topics spanning broad conceptual frameworks and specific circuit designs, and the future direction of synthetic biology is by no means limited to the few areas highlighted here. However, a unifying driving force in the field has been the desire to efficiently build biological systems, whether to improve our fundamental understanding of biology or to provide solutions for pressing global challenges. By developing conceptual frameworks and technical tools for the design, construction, and characterization of novel biological systems that can perform autonomous functions and interact with natural biological systems, synthetic biology is poised to transform our ability to probe, understand, and manipulate biology.

\section{Acknowledgements}

CDS is supported by funds from the National Institutes of Health, National Science Foundation, and Defense Advanced Research Projects Agency. YYC is supported by the Harvard University Society of Fellows.

\section{Author details}

'Society of Fellows, Harvard University, 78 Mount Auburn St, Cambridge, MA 02138, USA. ${ }^{2}$ Department of Systems Biology, Harvard Medical School, 200 Longwood Ave, WAB 536, Boston, MA 02115, USA. ${ }^{3}$ Division of Chemistry and 
Chemical Engineering, California Institute of Technology, 1200 East California Blvd, MC 210-41, Pasadena, CA 91125, USA. Department of Bioengineering, Stanford University, 473 Via Ortega, MC 4201, Stanford, CA 94305, USA

\section{Published: 20 February 2012}

\section{References}

1. Endy D: Foundations for engineering biology. Nature 2005, 438:449-453

2. Rebatchouk D, Daraselia N, Narita JO: NOMAD: a versatile strategy for in vitro DNA manipulation applied to promoter analysis and vector design. Proc Natl Acad Sci U S A 1996, 93:10891-10896.

3. Knight T: Idempotent Vector Design for Standard Assembly of Biobricks. MIT Synthetic Biology Working Group Technical Reports. Cambridge, MA: MIT; 2003.

4. Kouprina $\mathrm{N}$, Larionov $\mathrm{V}$ : Selective isolation of genomic loci from complex genomes by transformation-associated recombination cloning in the yeast Saccharomyces cerevisiae. Nat Protoc 2008, 3:371-377.

5. Gibson DG, Young L, Chuang RY, Venter JC, Hutchison CA, 3rd, Smith HO: Enzymatic assembly of DNA molecules up to several hundred kilobases. Nat Methods 2009, 6:343-345.

6. Canton B, Labno A, Endy D: Refinement and standardization of synthetic biological parts and devices. Nat Biotechno/ 2008, 26:787-793.

7. Tian J, Gong H, Sheng N, Zhou X, Gulari E, Gao X, Church G: Accurate multiplex gene synthesis from programmable DNA microchips. Nature 2004, 432:1050-1054.

8. LeProust EM, Peck BJ, Spirin K, McCuen HB, Moore B, Namsaraev E, Caruthers MH: Synthesis of high-quality libraries of long (150mer) oligonucleotides by a novel depurination controlled process. Nucleic Acids Res 2010, 38:2522-2540

9. Hughes RA, Miklos AE, Ellington AD: Gene synthesis: methods and applications. Methods Enzymol 2011, 498:277-309.

10. Gibson DG, Benders GA, Andrews-Pfannkoch C, Denisova EA, Baden-Tillson H, Zaveri J, Stockwell TB, Brownley A, Thomas DW, Algire MA, Merryman C, Young L, Noskov VN, Glass JI, Venter JC, Hutchison CA, Smith HO: Complete chemical synthesis, assembly, and cloning of a Mycoplasma genitalium genome. Science 2008, 319:1215-1220.

11. Kouprina N, Annab L, Graves J, Afshari C, Barrett JC, Resnick MA, Larionov V: Functional copies of a human gene can be directly isolated by transformation-associated recombination cloning with a small $3^{\prime}$ end target sequence. Proc Natl Acad Sci U S A 1998, 95:4469-4474.

12. Gibson DG, Smith HO, Hutchison CA 3rd, Venter JC, Merryman C: Chemical synthesis of the mouse mitochondrial genome. Nat Methods 2010, 7:901-903.

13. Gibson DG, Glass Jl, Lartigue C, Noskov VN, Chuang RY, Algire MA, Benders GA, Montague MG, Ma L, Moodie MM, Merryman C, Vashee S, Krishnakumar R, Assad-Garcia N, Andrews-Pfannkoch C, Denisova EA, Young L, Qi ZQ, Segall-Shapiro TH, Calvey CH, Parmar PP, Hutchison CA, Smith HO, Venter JC: Creation of a bacterial cell controlled by a chemically synthesized genome. Science 2010, 329:52-56.

14. Wang HH, Isaacs FJ, Carr PA, Sun ZZ, Xu G, Forest CR, Church GM: Programming cells by multiplex genome engineering and accelerated evolution. Nature 2009, 460:894-898.

15. Warner JR, Reeder PJ, Karimpour-Fard A, Woodruff LB, Gill RT: Rapid profiling of a microbial genome using mixtures of barcoded oligonucleotides. Nat Biotechnol 2010, 28:856-862.

16. Isaacs FJ, Carr PA, Wang HH, Lajoie MJ, Sterling B, Kraal L, Tolonen AC, Gianoulis TA, Goodman DB, Reppas NB, Emig CJ, Bang D, Hwang SJ, Jewett MC, Jacobson JM, Church GM: Precise manipulation of chromosomes in vivo enables genome-wide codon replacement. Science 2011, 333:348-353.

17. Dymond JS, Richardson SM, Coombes CE, Babatz T, Muller H, Annaluru N, Blake WJ, Schwerzmann JW, Dai J, Lindstrom DL, Boeke AC, Gottschling DE, Chandrasegaran S, Bader JS, Boeke JD: Synthetic chromosome arms function in yeast and generate phenotypic diversity by design. Nature 2011, 477:471-476

18. Purnick PE, Weiss $R$ : The second wave of synthetic biology: from modules to systems. Nat Rev Mol Cell Biol 2009, 10:410-422.

19. Alper H, Fischer C, Nevoigt E, Stephanopoulos G: Tuning genetic control through promoter engineering. Proc Natl Acad SciU S A 2005, 102:12678-12683

20. Ellis T, Wang X, Collins JJ: Diversity-based, model-guided construction of synthetic gene networks with predicted functions. Nat Biotechnol 2009,
27:465-471.

21. Karlsson M, Weber W, Fussenegger M: De novo design and construction of an inducible gene expression system in mammalian cells. Methods Enzymol 2011, 497:239-253.

22. Weber W, Rimann M, Spielmann M, Keller B, Daoud-El Baba M, Aubel D, Weber CC, Fussenegger M: Gas-inducible transgene expression in mammalian cells and mice. Nat Biotechno/ 2004, 22:1440-1444.

23. Levskaya A, Chevalier AA, Tabor JJ, Simpson ZB, Lavery LA, Levy M, Davidson EA, Scouras A, Ellington AD, Marcotte EM, Voigt CA: Synthetic biology: engineering Escherichia coli to see light. Nature 2005, 438:441-442.

24. Grilly C, Stricker J, Pang WL, Bennett MR, Hasty J: A synthetic gene network for tuning protein degradation in Saccharomyces cerevisiae. Mol Syst Biol 2007, 3:127.

25. Wong WW, Tsai TY, Liao JC: Single-cell zeroth-order protein degradation enhances the robustness of synthetic oscillator. Mol Syst Biol 2007, 3:130

26. Wu H, Hu Z, Liu XQ: Protein trans-splicing by a split intein encoded in a split DnaE gene of Synechocystis sp. PCC6803. Proc Natl Acad Sci U S A 1998, 95:9226-9231.

27. Muller J, Johnsson N: Split-ubiquitin and the split-protein sensors: chessman for the endgame. Chembiochem 2008, 9:2029-2038.

28. Win MN, Liang JC, Smolke CD: Frameworks for programming biological function through RNA parts and devices. Chem Biol 2009, 16:298-310.

29. Isaacs FJ, Dwyer DJ, Ding C, Pervouchine DD, Cantor CR, Collins JJ: Engineered riboregulators enable post-transcriptional control of gene expression. Nat Biotechnol 2004, 22:841-847.

30. Callura JM, Dwyer DJ, Isaacs FJ, Cantor CR, Collins JJ: Tracking, tuning, and terminating microbial physiology using synthetic riboregulators. Proc Natl Acad Sci U SA 2010, 107:15898-15903.

31. Win MN, Smolke CD: A modular and extensible RNA-based generegulatory platform for engineering cellular function. Proc Natl Acad SCi US A 2007, 104:14283-14288.

32. An Cl, Trinh VB, Yokobayashi Y: Artificial control of gene expression in mammalian cells by modulating RNA interference through aptamer-small molecule interaction. RNA 2006, 12:710-716.

33. Beisel CL, Chen YY, Culler SJ, Hoff KG, Smolke CD: Design of small moleculeresponsive microRNAs based on structural requirements for Drosha processing. Nucleic Acids Res 2010, 39:2981-2994.

34. Sinha J, Reyes SJ, Gallivan JP: Reprogramming bacteria to seek and destroy an herbicide. Nat Chem Biol 2010, 6:464-470.

35. Culler SJ, Hoff KG, Smolke CD: Reprogramming cellular behavior with RNA controllers responsive to endogenous proteins. Science 2010, 330:1251-1255.

36. Good MC, Zalatan JG, Lim WA: Scaffold proteins: hubs for controlling the flow of cellular information. Science 2011, 332:680-686.

37. Bhattacharyya RP, Remenyi A, Good MC, Bashor CJ, Falick AM, Lim WA: The Ste5 scaffold allosterically modulates signaling output of the yeast mating pathway. Science 2006, 311:822-826.

38. Bashor CJ, Helman NC, Yan S, Lim WA: Using engineered scaffold interactions to reshape MAP kinase pathway signaling dynamics. Science 2008, 319:1539-1543

39. Dueber JE, Wu GC, Malmirchegini GR, Moon TS, Petzold CJ, Ullal AV, Prather $\mathrm{KL}$, Keasling JD: Synthetic protein scaffolds provide modular control over metabolic flux. Nat Biotechnol 2009, 27:753-759.

40. Delebecque $\mathrm{CJ}$, Lindner $\mathrm{AB}$, Silver PA, Aldaye FA: Organization of intracellular reactions with rationally designed RNA assemblies. Science 2011, 333:470-474

41. Hasty J, Dolnik M, Rottschafer V, Collins JJ: Synthetic gene network for entraining and amplifying cellular oscillations. Phys Rev Lett 2002, 88:148101.

42. Stricker J, Cookson S, Bennett MR, Mather WH, Tsimring LS, Hasty J: A fast, robust and tunable synthetic gene oscillator. Nature 2008, 456:516-519.

43. Kramer BP, Fussenegger M: Hysteresis in a synthetic mammalian gene network. Proc Natl Acad Sci U S A 2005, 102:9517-9522.

44. Elowitz MB, Leibler S: A synthetic oscillatory network of transcriptional regulators. Nature 2000, 403:335-338.

45. Hasty J, McMillen D, Collins JJ: Engineered gene circuits. Nature 2002 420:224-230

46. Rosenfeld N, Elowitz MB, Alon U: Negative autoregulation speeds the response times of transcription networks. J Mol Biol 2002, 323:785-793.

47. Eldar A, Elowitz MB: Functional roles for noise in genetic circuits. Nature 2010, 467:167-173. 
48. Gardner TS, Cantor CR, Collins JJ: Construction of a genetic toggle switch in Escherichia coli. Nature 2000, 403:339-342.

49. Tigges M, Marquez-Lago TT, Stelling J, Fussenegger M: A tunable synthetic mammalian oscillator. Nature 2009, 457:309-312

50. Tamsir A, Tabor JJ, Voigt CA: Robust multicellular computing using genetically encoded NOR gates and chemical 'wires'. Nature 2011, 469:212-215.

51. Win MN, Smolke CD: Higher-order cellular information processing with synthetic RNA devices. Science 2008, 322:456-460.

52. Rinaudo K, Bleris L, Maddamsetti R, Subramanian S, Weiss R, Benenson Y: A universal RNAi-based logic evaluator that operates in mammalian cells. Nat Biotechnol 2007, 25:795-801.

53. Friedland AE, Lu TK, Wang X, Shi D, Church G, Collins JJ: Synthetic gene networks that count. Science 2009, 324:1199-1202.

54. Kramer BP, Viretta AU, Daoud-El-Baba M, Aubel D, Weber W, Fussenegger M: An engineered epigenetic transgene switch in mammalian cells. Nat Biotechnol 2004, 22:867-870.

55. Ajo-Franklin CM, Drubin DA, Eskin JA, Gee EP, Landgraf D, Phillips I, Silver PA Rational design of memory in eukaryotic cells. Genes Dev 2007, 21:2271-2276

56. Deans TL, Cantor CR, Collins JJ: A tunable genetic switch based on RNAi and repressor proteins for regulating gene expression in mammalian cells. Cell 2007, 130:363-372

57. Xie Z, Wroblewska L, Prochazka L, Weiss R, Benenson Y: Multi-input RNAibased logic circuit for identification of specific cancer cells. Science 2011, 333:1307-1311.

58. You L, Cox RS 3rd, Weiss R, Arnold FH: Programmed population control by cell-cell communication and regulated killing. Nature 2004, 428:868-871.

59. Basu S, Gerchman Y, Collins CH, Arnold FH, Weiss R: A synthetic multicellular system for programmed pattern formation. Nature 2005, 434:1130-1134

60. Brenner K, Arnold FH: Self-organization, layered structure, and aggregation enhance persistence of a synthetic biofilm consortium. PLoS One 2011, 6:e16791.

61. Brenner K, Karig DK, Weiss R, Arnold FH: Engineered bidirectional communication mediates a consensus in a microbial biofilm consortium. Proc Natl Acad Sci U S A 2007, 104:17300-17304

62. Danino T, Mondragon-Palomino O, Tsimring L, Hasty J: A synchronized quorum of genetic clocks. Nature 2010, 463:326-330.

63. Tabor JJ, Salis HM, Simpson ZB, Chevalier AA, Levskaya A, Marcotte EM, Voigt CA, Ellington AD: A synthetic genetic edge detection program. Cell 2009, 137:1272-1281

64. Weber W, Daoud-El Baba M, Fussenegger M: Synthetic ecosystems based on airborne inter- and intrakingdom communication. Proc Natl Acad Sci U S A 2007, 104:10435-10440.

65. Weber W, Schuetz M, Denervaud N, Fussenegger M: A synthetic metabolitebased mammalian inter-cell signaling system. Mol Biosyst 2009, 5:757-763.

66. Kobayashi H, Kaern M, Araki M, Chung K, Gardner TS, Cantor CR, Collins JJ: Programmable cells: interfacing natural and engineered gene networks. Proc Natl Acad SciU S A 2004, 101:8414-8419.

67. Burrill DR, Silver PA: Synthetic circuit identifies subpopulations with sustained memory of DNA damage. Genes Dev 2011, 25:434-439.

68. Kemmer C, Gitzinger M, Daoud-El Baba M, Djonov V, Stelling J, Fussenegger M: Self-sufficient control of urate homeostasis in mice by a synthetic circuit. Nat Biotechnol 2010, 28:355-360

69. Kemmer C, Fluri DA, Witschi U, Passeraub A, Gutzwiller A, Fussenegger M: A designer network coordinating bovine artificial insemination by ovulation-triggered release of implanted sperms. J Control Release 2011, 150:23-29.

70. Nandagopal N, Elowitz MB: Synthetic biology: integrated gene circuits. Science 2011, 333:1244-1248.

71. Cagatay T, Turcotte M, Elowitz MB, Garcia-Ojalvo J, Suel GM: Architecturedependent noise discriminates functionally analogous differentiation circuits. Cell 2009, 139:512-522

72. Mody A, Weiner J, Ramanathan S: Modularity of MAP kinases allows deformation of their signalling pathways. Nat Cell Biol 2009, 11:484-491.

73. O'Shaughnessy EC, Palani S, Collins JJ, Sarkar CA: Tunable signal processing in synthetic MAP kinase cascades. Cell 2011, 144:119-131.

74. Sprinzak D, Lakhanpal A, Lebon L, Santat LA, Fontes ME, Anderson GA, GarciaOjalvo J, Elowitz MB: Cis-interactions between Notch and Delta generate mutually exclusive signalling states. Nature 2010, 465:86-90.
75. Naesby M, Nielsen SV, Nielsen CA, Green T, Tange TO, Simón E, Knechtle P, Hansson A, Schwab MS, Titiz O, Folly C, Archila RE, Maver M, van Sint Fiet S, Boussemghoune T, Janes M, Kumar AS, Sonkar SP, Mitra PP, Benjamin VA, Korrapati N, Suman I, Hansen EH, Thybo T, Goldsmith N, Sorensen AS: Yeast artificial chromosomes employed for random assembly of biosynthetic pathways and production of diverse compounds in Saccharomyces cerevisiae. Microb Cell Fact 2009, 8:45.

76. Shao Z, Zhao H: DNA assembler, an in vivo genetic method for rapid construction of biochemical pathways. Nucleic Acids Res 2009, 37:e16.

77. Shao Z, Luo Y, Zhao H: Rapid characterization and engineering of natural product biosynthetic pathways via DNA assembler. Mol Biosyst 2011, 7:1056-1059.

78. Pfleger BF, Pitera DJ, Smolke CD, Keasling JD: Combinatorial engineering of intergenic regions in operons tunes expression of multiple genes. Nat Biotechnol 2006, 24:1027-1032

79. Babiskin AH, Smolke CD: Synthetic RNA modules for fine-tuning gene expression levels in yeast by modulating RNase III activity. Nucleic Acids Res 2011, 39:8651-8664.

80. Babiskin AH, Smolke CD: A synthetic library of RNA control modules for predictable tuning of gene expression in yeast. Mol Syst Bio/ 2011, 7:471.

81. Han J, McLane B, Kim EH, Yoon JW, Jun HS: Remission of diabetes by insulin gene therapy using a hepatocyte-specific and glucose-responsive synthetic promoter. Mol Ther 2011, 19:470-478.

82. Ye H, Daoud-El Baba M, Peng RW, Fussenegger M: A synthetic optogenetic transcription device enhances blood-glucose homeostasis in mice. Science 2011, 332:1565-1568

83. Gitzinger M, Kemmer C, El-Baba MD, Weber W, Fussenegger M: Controlling transgene expression in subcutaneous implants using a skin lotion containing the apple metabolite phloretin. Proc Natl Acad Sci U S A 2009, 106:10638-10643.

84. Chen YY, Jensen MC, Smolke CD: Genetic control of mammalian T-cell proliferation with synthetic RNA regulatory systems. Proc Natl Acad Sci U S A 2010, 107:8531-8536.

85. Hannink N, Rosser SJ, French CE, Basran A, Murray JA, Nicklin S, Bruce NC: Phytodetoxification of TNT by transgenic plants expressing a bacterial nitroreductase. Nat Biotechnol 2001, 19:1168-1172.

86. Singh BK, Walker A: Microbial degradation of organophosphorus compounds. FEMS Microbiol Rev 2006, 30:428-471.

87. Gadd GM: Metals, minerals and microbes: geomicrobiology and bioremediation. Microbiology 2010, 156:609-643.

88. Antunes MS, Morey KJ, Smith JJ, Albrecht KD, Bowen TA, Zdunek JK, Troupe JF, Cuneo MJ, Webb CT, Hellinga HW, Medford Jl: Programmable ligand detection system in plants through a synthetic signal transduction pathway. PLoS One 2011, 6:e16292.

89. Schuldiner M, Itskovitz-Eldor J, Benvenisty N: Selective ablation of human embryonic stem cells expressing a "suicide" gene. Stem Cells 2003, 21:257-265.

90. Maherali N, Ahfeldt T, Rigamonti A, Utikal J, Cowan C, Hochedlinger K: A high-efficiency system for the generation and study of human induced pluripotent stem cells. Cell Stem Cell 2008, 3:340-345.

91. Kueh HY, Rothenberg EV: Regulatory gene network circuits underlying T cell development from multipotent progenitors. Wiley Interdiscip Rev Syst Biol Med 2012, 4:79-102.

92. Thomson M, Liu SJ, Zou LN, Smith Z, Meissner A, Ramanathan S: Pluripotency factors in embryonic stem cells regulate differentiation into germ layers. Cell 2011, 145:875-889.

93. Conrado RJ, Wu GC, Boock JT, Xu H, Chen SY, Lebar T, Turnsek J, Tomsic N, Avbelj M, Gaber R, KoprivnjakT, Mori J, Glavnik V, Vovk I, Bencina M, HodnikV, Anderluh G, Dueber JE, Jerala R, Delisa MP: DNA-guided assembly of biosynthetic pathways promotes improved catalytic efficiency. Nucleic Acids Res 2011. doi: 10.1093/nar/gkr888

doi:10.1186/gb-2012-13-2-240

Cite this article as: Chen YY, et al:: Synthetic biology: advancing biological frontiers by building synthetic systems. Genome Biology 2012, 13:240. 